\title{
LES INCLUSIONS ROCHEUSES DANS LES GLACIERS
}

\author{
JEAN PIERRE PORTMANN
}

A. GENERALITES

\author{
"We need still more facts, more studies of the \\ structure of glaciers.》
}

(Hellingworth, 195o, p. 431)

Les présentes recherches découlent de la constatation qu'un glacier est un système complexe comprenant des fragments rocheux de toutes dimensions pris dans la masse interstitielle qu'est la glace. La phase glace est en général bien étudiée alors que la phase discontinue, comprenant les inclusions rocheuses et la seule subsistant après la fonte, est négligée.

Bien que l'inlandsis groenlandais, par exemple, soit parfois qualifié de pauvre en matériel erratique, la présence de débris rocheux dans la zone marginale, soumise à l'ablation, est attestée (J.P. Koch et A. Wegener, 1912, fig. 42; H. G. Backlund, 1931, fig. 3 ; Drygalski et Machatscheck, 1942, pl. 7). D'après Ward (1954, p. 11), les inclusions constituent quelques \%, en volume, de la glace; d'après les estimations de Lewis (1950, p. 434), le $95 \%$ de l'épaisseur de la falaise bordière de l'inlandsis consiste en glace. Dans le cas du Barnes Ice Cap (East Central Baffin Land), les plans de schistosité renfermant des débris rocheux sont confinés à la périphérie dans une zone correspondant à peu près à $1 \%$ de la surface totale.

Il est probable que la teneur, même faible, en matériel erratique puisse modifier les caractéristiques physiques de la glace. La plasticité, le mode de déformation et l'intensité de l'ablation en sont en tout cas influencés.

L'ablation, par exemple, est assurément plus élevée dans les zones riches en matériel pierreux. Goldthwait publie des estimations rigoureuses à ce propos (1951, p. 571); ainsi pour le Barnes Ice Cap, l'ablation hebdomadaire serait de $20,3 \mathrm{~cm}$ pour la glace claire, pure, et de $33 \mathrm{~cm}$ pour la glace recouverte d'une mince couche de debris rocheux, ce qui correspondrait à une ablation annuelle s'élevant respectivement à $101,6-152,4 \mathrm{~cm}$ et à $152,4-203,3 \mathrm{~cm}$. D'après WARD (1952, p. 12) «the different rates of melting of glacier ice and snow in association with rock debris present a fascinating study in the exchange of heat between the surface and the atmosphere, and, in conjuction with the ice sherring, they form the basis of movement and construction of ablation moraines». Cet auteur précise que la réflexion de la neige humide est de 0,6 ; celle de la glace pure et humide 0,4 , alors que pour les débris rocheux les mesures varient entre 0,1 et 0,4 suivant la couleur. Il mentionne encore que souvent la température des roches est supérieure à la température de l'air et que, parfois même, la température de l'air, au-dessus des débris de roches, est supérieure de $2^{\circ}$ à celle au-dessus de la glace pure, à $70 \mathrm{~m}$ de distance. Les valeurs citées par WASHBURN (1956) ne font que confirmer ce qui précède. On fera remarquer encore que la présence de roches dans la glace en favorise la fonte par l'augmentation de la porosité provoquant une circulation plus intense des eaux intraglaciaires. Ceci est le cas, en particulier, dans la portion basale du glacier, enrichie en fragments rocheux de toutes dimensions. A ce point de vue, plusieurs minces couches de fins débris seront équivalentes à une intercalation épaisse de fragments plus grossiers. En surface, il arrive aussi qu'une assise de débris rocheux retarde l'ablation des masses sous-jacentes.

L'influence des inclusions rocheuses dans la déformation de l'ensemble de la masse de glace nous paraît due à leur répartition plutôt qu'à leur abondance. En effet, la répartition n'est pas uniforme: Leighton (1951) a rappelé leur concentration le long des ogives, remarquant en outre que les éléments grossiers sont localisés sur certains plans (p. 581). Les observations de Goldthwait (1951) apportent des données précises sur la dispersion des débris pierreux dans le Barnes Ice Cap (Baffin Land); ceux-ci, présentant les dimensions les plus diverses, sont concentrés le long de plans de cisaillement, en lits d'épaisseur variable séparés par de la glace qui en est pauvre ou dépourvue.

Actuellement, nous ne disposons que d'observations vagues et fragmentaires; seules des études systématiques, fournissant des valeurs numériques, permettront des considérations valables sur un certain nombre de problèmes. Il importerait, par exemple, de préciser la proportion des fragments rocheux, leur répartition, leur concentration le long de certains plans sédimentaires et structuraux.

La fréquence des constituants en fonction de leur dimension permettrait éventuellement la mise en évidence d'un spectre granulométrique, caractéristique du transport glaciaire pour des espèces pétrographiques déterminées. 


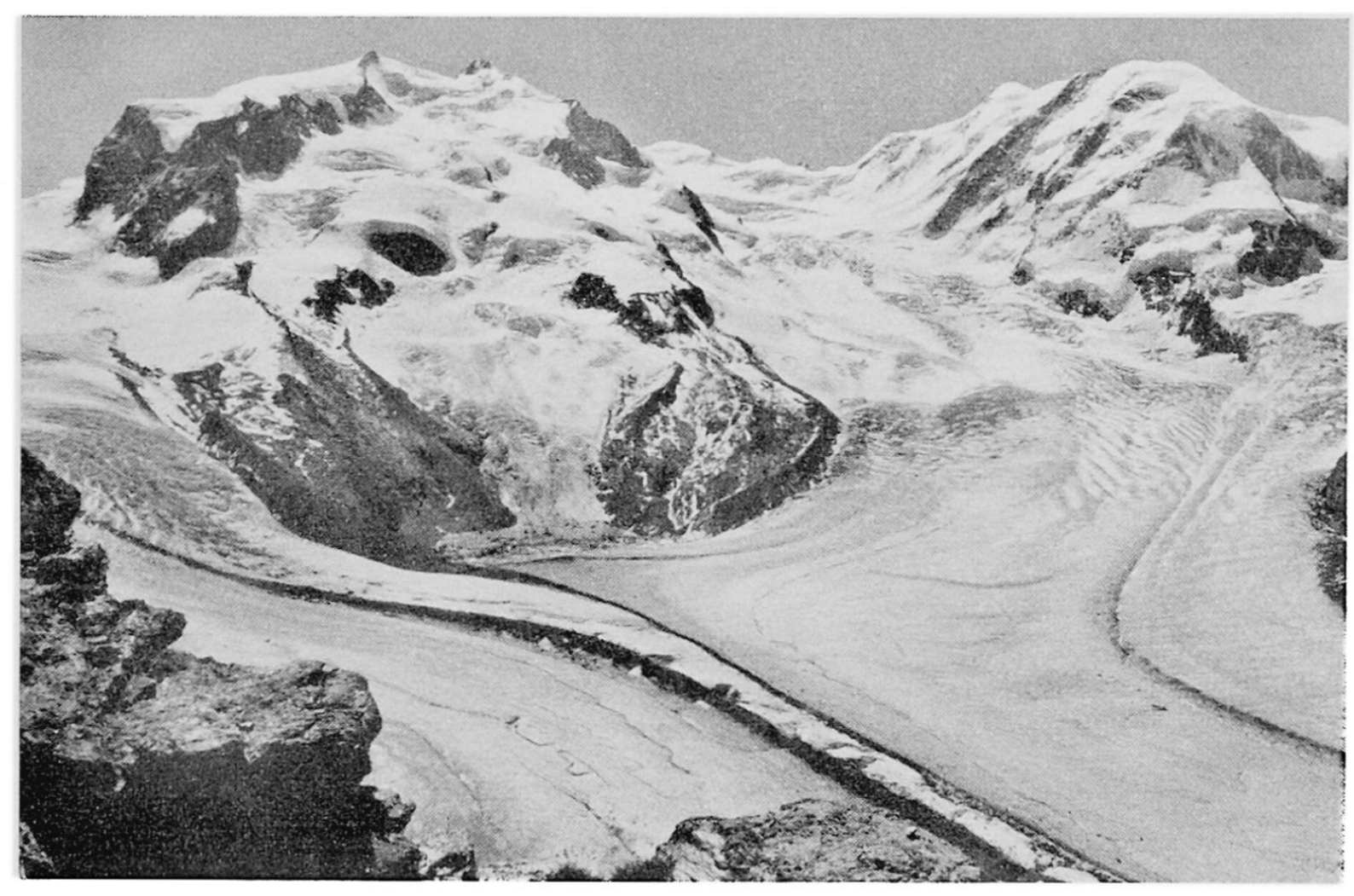

Fig. 1 Du Gornergrat en direction du Mt Rose et du Lyskamm (Photo O. Furter)

L'identification minéralogique et pétrographique des inclusions de la glace renseignerait sur leur provenance, le chemin parcouru, le mode de dispersion et, dans certains cas même, donnerait des indications sur la nature du socle rocheux enfoui sous la glace (cas des inlandsis).

Quant à la forme des éléments rocheux, elle fournirait des précisions sur le mode d'usure, sur la longueur du transport glaciaire.

Enfin, l'orientation des galets, en relation avec la direction d'écoulement de la masse de glace, permettrait des considérations d'ordre cinématique (GLEN, 1956). Ajoutons que des mesures sur la structure des glaciers (crevasses, plans de schistosité etc.) sont à préconiser en complément de celles mentionnées ci-dessus. Les observations publiées par MEIER, RigsBy, SHARP (1954) et par Lliboutry (1958) en montrent la portée générale.

Toutes les recherches sur les inclusions rocheuses dans les glaciers et les inlandsis permettront de comprendre la genèse des dépôts glaciaires actuels et anciens, ce qui ne peut que contribuer à l'enrichissement de nos connaissances sur le Quaternairc. Les observations sommaires consignées ci-dessous dérivent d'une première tentative d'étudier les inclusions rocheuses dans un glacier.

\section{B. LE GLACIER DE GORNER SUR ZERMATT (SUISSE) ${ }^{1}$}

«Pour nous qui venions les visiter tout exprès pour les interroger sur leur nature intime et sur le rôlc qu'clles ont joué dans l'histoire de la terre, ces masses de glace devaient nous apparaitre sous le double charme de la beauté pittoresque et de l'intérêt scientifique.»

(E. DESOR, 1844, p. 67)

«Le Glacier de Gorner est le principal appareil glaciaire de la haute vallée de St-Nicolas (Valais). Sa superficie de $67 \mathrm{~km} 2$ lui assigne la deuxième place parmi les glaciers suisses... D'une longueur totale de $15 \mathrm{~km}$ environ, le Glacier de Gorner s'écoule de l'Est à l'Ouest, soit du Massif du Mont Rose (4638 m), le plus élevé de Suisse, vers Zermatt (1611 m) ... Huit affluents composent cette grande masse glaciaire dont la partie inférieure, qui porte le nom de

1 Carte Nationale de la Suisse: Feuille normale 568, Mischabel-W. 
Bodengletscher, se termine vers $2000 \mathrm{~m}$ dans une région sauvage et escarpée à $3 \mathrm{~km}$ en amont de Zermatt environ» (Renaud, 1950).

Ces glaciers accusent le caractère du climat régional. La limite inférieure des neiges persistantes s'y élève à plus de $3200 \mathrm{~m}$. Depuis 1889 , le Glacier de Gorner manifeste une décrue continue se marquant par un abaissement annuel moyen de la surface du glacier d'environ $1,5 \mathrm{~m}$ entre 2400 et $2600 \mathrm{~m}$. Les mesures de vitesses superficielles ont montré que si le maximum de l'avancement se situe un peu au-dessous de la limite du névé, les valeurs varient par contre énormément d'un affluent à l'autre. Elles paraissent révéler que le Glacier de Grenz est réellement le bras principal du glacier composé.

\section{Orientation des galets à la surface du glacier ${ }^{2}$}

Nous avons mesuré l'orientation des galets en une quinzaine d'endroits répartis de part et d'autre de la courbe de niveau de $2660 \mathrm{~m}$; plus exactement, à partir de $2800 \mathrm{~m}$ jusque vers $2640 \mathrm{~m}$ pour les glaciers de Gorner et de Grenz, c'est-à-dire jusque peu au-dessous de la zone de leur confluence. Le plus souvent possible, les mesures furent faites là où la glace présentait une surface plus ou moins horizontale et où, en tout cas, aucune trace de ruissellement n'était visible. Seuls les galets allongés, de $20 \mathrm{~cm}$ environ, et encore enchassés dans la glace furent pris en considération. Parfois la rareté du matériel et le manque de temps nous contraignirent à ne relever l'orientation que de 50 galets par station.

Les diagrammes représentant nos mesures mettent en évidence une nette orientation préférentielle, parfois avec un seul maximum, souvent avec deux maximums. La figure 2 représente quelques-uns de ces diagrammes. Lorsque deux maximums existent, l'angle qu'ils forment est de $50^{\circ}$ en moyenne, les variations s'étendant de $32^{\circ}$ à $86^{\circ}$. Les blocs, eux aussi, peuvent montrer une orientation préférentielle.

Les orientations des galets allongés, englobés dans la glace superficielle, ont été confrontées avec les mesures d'écoulement en surface effectuées par RENAUD (1950) et BAuER (1955). Dans quelques cas, ces deux catégories de directions sont ou paral-
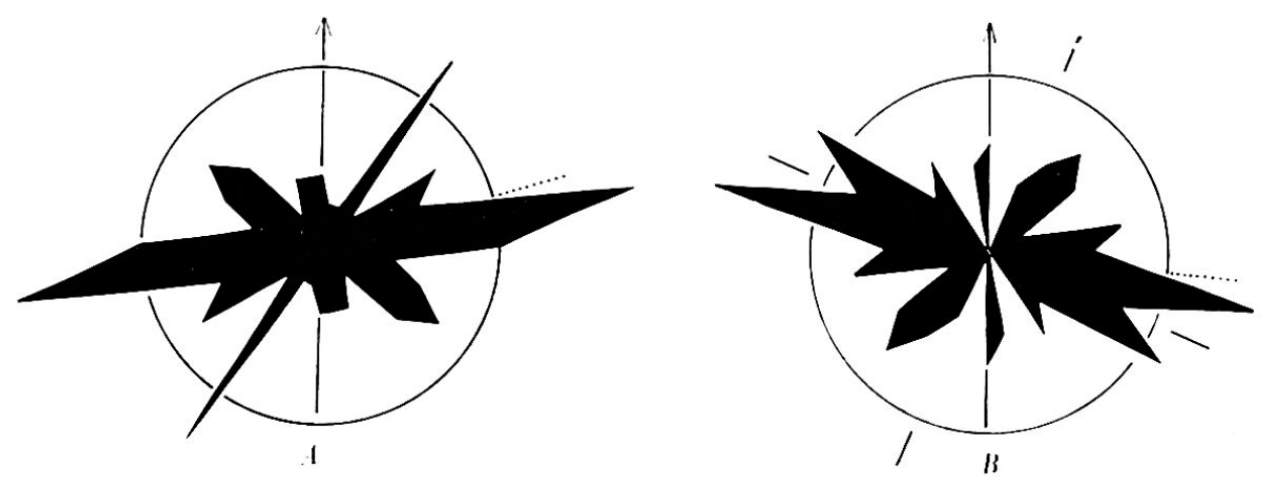

Fig. 2

Orientation des galets $(A, B, C)$ et des blocs (D) à la surface du glacier.

(La circonférence correspond à $10 \% ;=$ : crête morainique ou alignement de blocs; I: crevasses, plans structuraux; ...: médiane, direction à $50 \%$ )
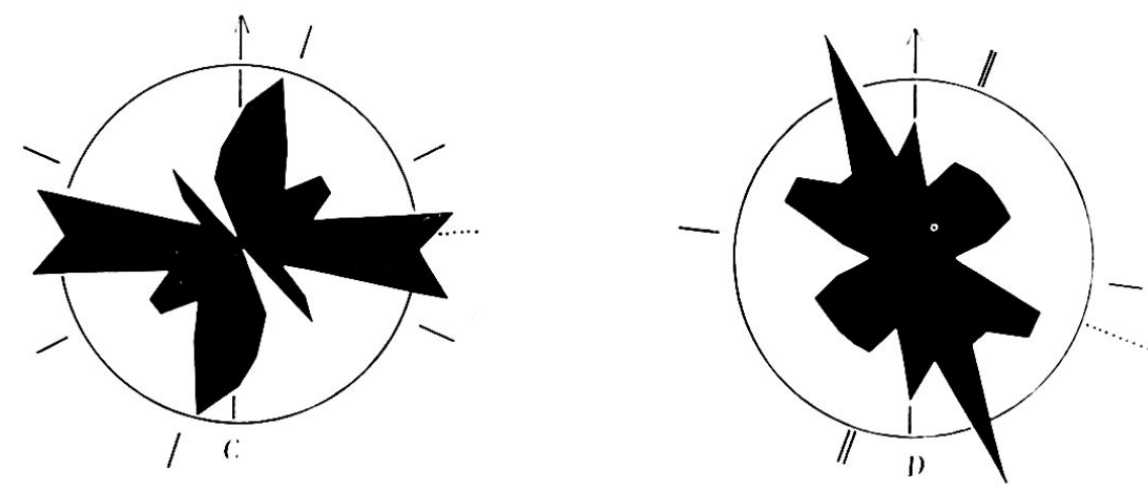

2 Ces mesures furent effectuées du 13 au 20 août 1956. Nous tenons à remercier la Commission des Glaciers de la Société helvétique des sciences naturelles pour son subside qui couvrit les frais de voyage et de séjour à la Cabane Bétemp (Cabanc du Mont Rose). 


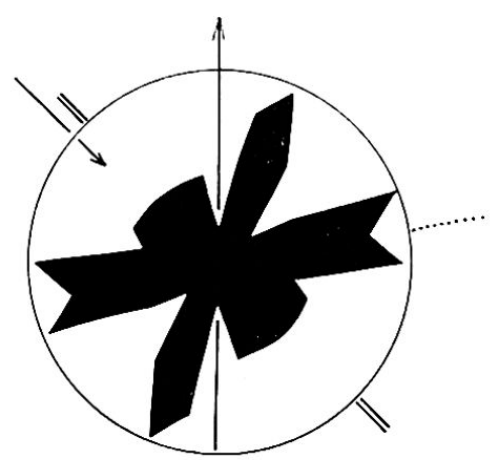

$A$

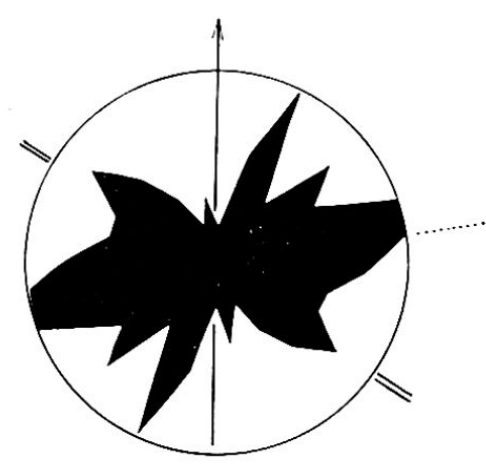

B
Fig. 3! Orientation des galets allongés dans deux moraines; A) Moraine latérale droite du glacier de Grenz,

B) Moraine latérale gauche du glacier du Mt. Rose.

$(\rightarrow$ : stries; $=$ : crête morainique; .... médiane, direction à $50 \%$; la circonférence correspond à $10 \%$ )

lèles ou perpendiculaires mais, en général, elles se coupent irrégulièrement; il en est de même avec les plans structuraux du glacier (crevasses, schistosité).

La figure 4 représente l'ensemble des observations; les directions d'écoulement sont indiquées par des flèches, les crevasses par des traits interrompus, les plans de schistosité et les autres plans structuraux par des traits pleins. Les circonférences désignent les endroits où les mesures d'orientation des galets allongés furent effectuées; les rhombes correspondent à des maximums très nets, les traits à des maximums secondaires, moins marqués.

On remarque que les directions d'écoulement correspondent en gros à ce qui peut être déduit de la morphologie générale; on notera les directions inattendues relevées dans le secteur à l'aval du lac du Mont-Rose. Les crevasses sont transversales ou subtransversales au courant glaciaire, c'est-à-dire plus ou moins perpendiculaires aux marges du glacier; elles présentent des variations relativement faibles dues aux conditions locales (topographie sous glaciaire, vitesse d'écoulement etc.). Quant aux plans longitudinaux, plans de schistosité, ils manifestent une constance évidente dans leur orientation. Celle-ci ne coïncide d'ailleurs pas toujours avec la direction d'écoulement superficiel et n'est pas forcément perpendiculaire aux crevasses, bien que cela soit le cas en plusieurs endroits; de plus, elle ne suit pas la direction des accumulations morainiques. Ces plans longitudinaux sont en général verticaux; on remarquera néanmoins le plongement particulier de deux d'entre eux sur la transversale Äuss Gornerli Schwärze, plongement dirigé des deux zones bordières vers la partie centrale du glacier. L'absence de mesure, de part et d'autre de la moraine médiane, nous empêche de savoir s'il s'agit vraiment d'une disposition synclinale s'étendant sur toute la largeur du complexe glaciaire. De même, à propos des plongements mesurés plus en aval, au-dessous de la courbe de $2600 \mathrm{~m}$; là, les plans orientés longitudinalement semblent aussi dessiner un synclinal, large de $400 \mathrm{~m}$, bordé au nord par un pli anticlinal, asymétrique, visible d'ailleurs $1 \mathrm{~km}$ plus en aval dans la même direction (sous le Riffelhorn).

Si l'orientation préférentielle des galets allongés est évidente, les relations avec les différents plans mentionnés ci-dessus sont souvent peu claires et variables. Plusieurs diagrammes présentent des maximums orientés dans une même direction; certaines orientations se répètent donc, parfois perpendiculaires aux crevasses voisines ou correspondant quelquefois à la bissectrice de l'angle formé par ces dernières et les plans longitudinaux. Les mesures dont nous disposons ne sont pas suffisamment nombreuses pour que nous puissions nous hasarder à formuler des constatations générales; nous nous bornerons à rcmarquer que les maximums mis en évidence présentent une régularité indéniable dans leur orientation, en accord semble-t-il avec la physiographie glaciaire.

\section{Orientation des galets dans trois moraines marginales}

Désireux de poursuivre les recherches commencées en 1952, au glacier de Moiry (Portmann, 1956), nous avons relevé l'orientation des galets allongés dans trois mo- 


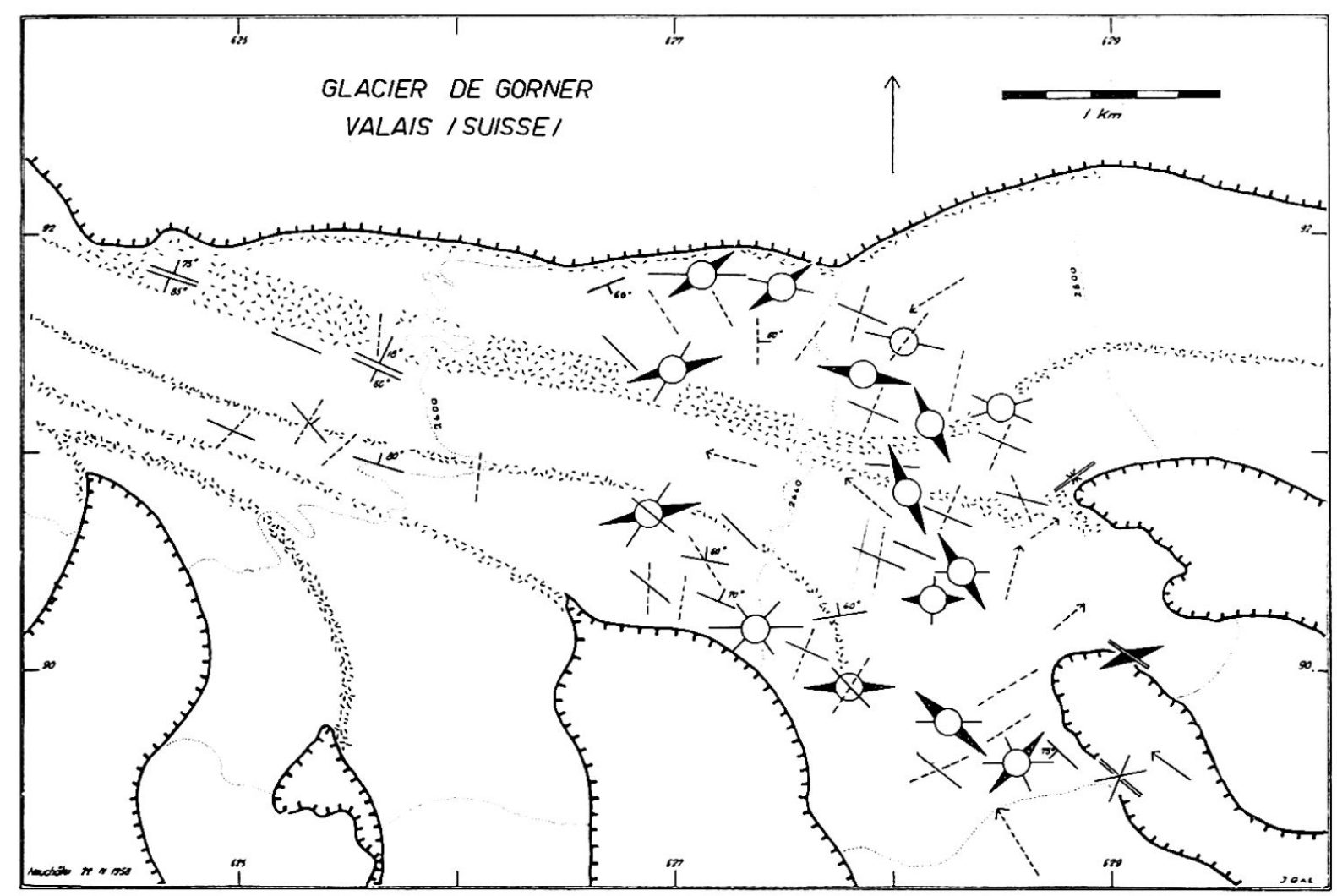

Fig. 4 Carte d'une portion du glacier de Gorner et de ses affluents. Plans structuraux du glacier et orientation préférentielle des galets allongés.

(Voir texte p. 4)

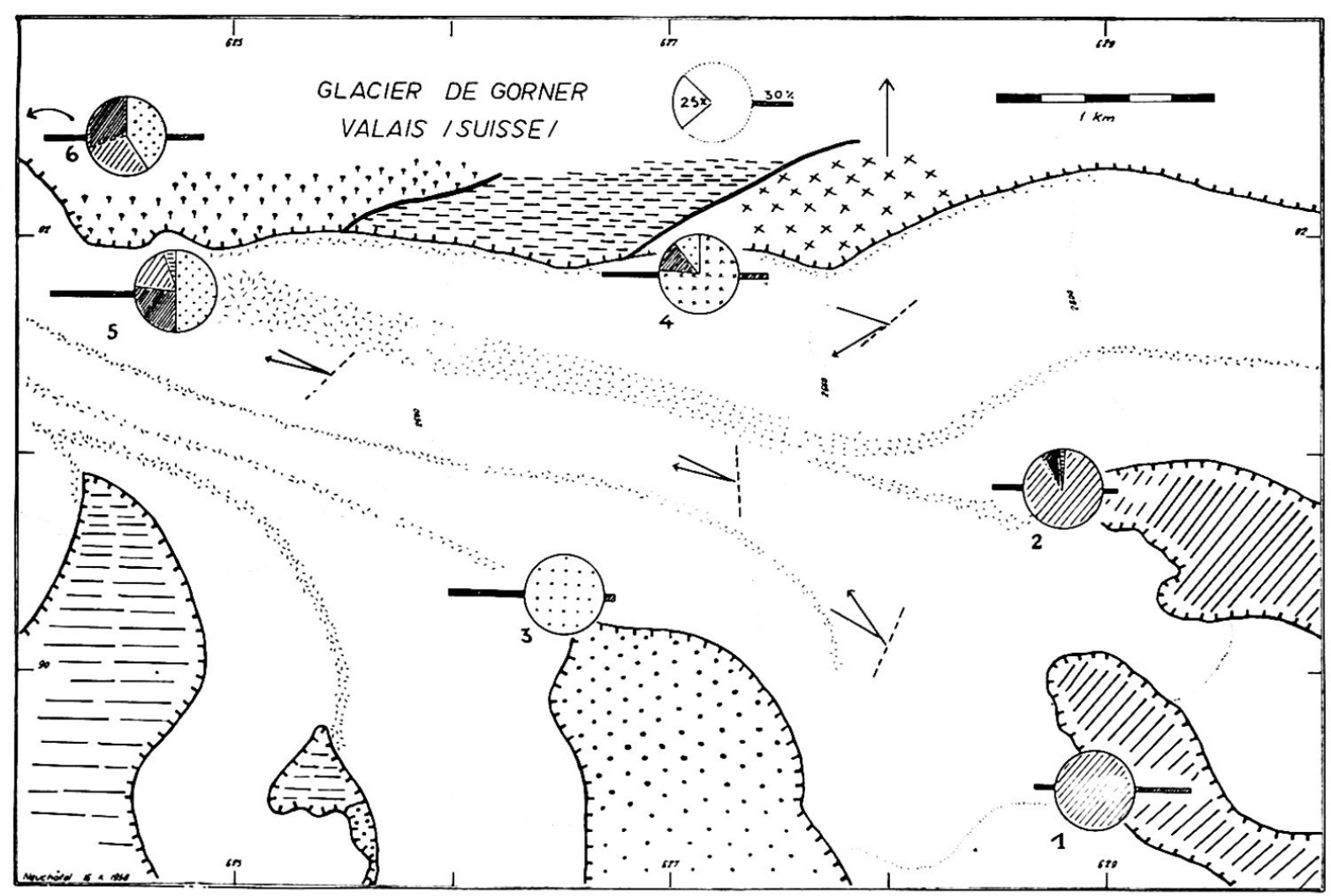

Fig. 5 Carte d'une portion du glacier de Gorner et de ses affluents. Pétrographie des moraines. 

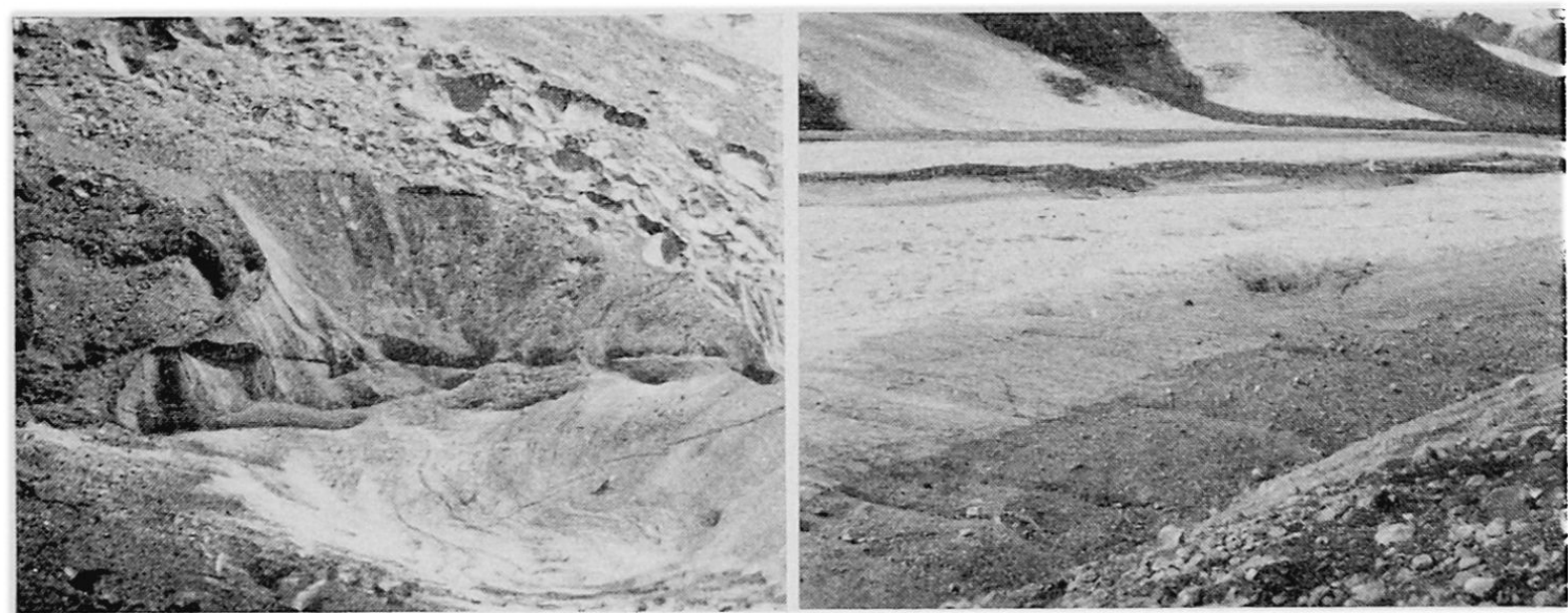

Fig: 6 et 7 A gauche: Langue terminale du glacier de Boden (plans de cisaillement)

A droite: Glacier de Gorner (Aeuß. Gornerli) En bordure: zone riche en fragments rocheux et entonnoir (Renaud, 1936)

Photo J. P. Portmann, 1956

raines marginales. En premier lieu dans la moraine latérale droite du glacier de Grenz, à Untere Plattje (fig. 3); deuxièmement dans la moraine latérale gauche du glacier du Mt-Rose, au NE d'Untere Plattje, troisièmement dans la moraine latérale gauche du glacier de Gorner (NW Ob dem See).

Aux deux premiers endroits seulement, l'orientation préférentielle est manifeste, subperpendiculaire à la direction de la crête morainique, elle-même parallèle aux stries relevées sur les roches voisines.

\section{Pétrographie de quelques moraines}

Six échantillons, prélevés dans les moraines du bassin glaciaire de Gorner, ont été tamisés et leurs constituants identifiés pétrographiquement. Les résultats mettent en évidence les relations, d'une part, entre la nature des roches en place et des moraines et, d'autre part, entre le spectre granulométrique et la lithologie de ces dernières. Ces résultats montrent aussi la dispersion des fragments rocheux à partir des gisements in situ. Ces investigations préliminaires donnent un aperçu des recherches systématiques qui pourraient être entreprises, sur les glaciers actuels et en bordure de ceux-ci, sur le transport et la répartition des minéraux et des roches (minéraux lourds, minéraux caractéristiques, morphométrie et morphogenèse des fragments rocheux, par exemple).

Le glacier de Gorner et ses affluents s'étalent au cœur des Alpes penniques, dans la zone nord-occidentale de la Nappe $d u M t$-Rose et du Pennique mésozoïque séparant cette dernière de la Nappe de la Dent Blanche, unité supérieure, qui s'étend plus à l'ouest. Pétrographiquement, les formations encadrant le bassin glaciaire peuvent être brièvement caractérisées comme suit d'après les levés de P. Beartri (1953). Ainsi que le montre la figure 5, la partie amont de la rive droite du glacier de Gorner est constituée du vieux cristallin de la Nappe du Mt-Rose comprenant des schistes métamorphiques (micaschistes à grenat, schistes à muscovite) représentés sur la carte par des croix. Plus en aval apparaissent les formations triasiques de la zone sédimentaire du Gornergrat; on y rencontre en particulier des quartzites plaquetés (traits horizontaux). Plus bas se dresse le massif de serpentine du Riffelhorn (ponctuations caudées). Quant à la rive sud, on a affaires dans les promotoires de Plattje et d'Ob dem See, contreforts du Mt-Rose, à des granites plutôt grossiers, à des gneiss oeillés et d'autres variétés gneissiques (hachures obliques). La Schwärze, formant la rive gauche du glacier de Grenz, est constituée d'ophiolithes, surtout de prasinites et d'amphibolites (ponctuations). De là jusqu'au glacier de Théodule, affleure la partie méridionale de la zone 
sédimentaire du Gornergrat; comprenant ici des schistes lustrés, des quartzites à muscovite, parfois granatifères, toutes formations considérées comme jurassiques (traits horizontaux).

Le tableau ci-dessous renseigne sur la granulométrie de la masse fine, inférieure à $20 \mathrm{~mm}$, des moraines latérales. Sur la figure 5, à gauche de chaque cercle représentant la pétrographie des échantillons, nous avons indiqué le pourcentage en granules d'un diamètre supérieur à $2 \mathrm{~mm}$; le trait dessiné à droite du cercle se rapporte aux éléments fins, plus petits que $0,2 \mathrm{~mm}$.

Quant à la pétrographie des divers échantillons, elle reflète celle des roches en place voisines. Pour ces recensements, nous ne nous en sommes tenu qu'aux fragments de 6 à $20 \mathrm{~mm}$ de diamètre, les répartissant entre les diverses subdivisions lithologiques mentionnées dans la description ci-dessus. Nous avons utilisé les mêmes signatures, établissant une distinction entre les gneiss clairs (hachures espacées) et les gneiss foncés (hachures serrées). Il est évident que seule une identification lithologique très précise donnerait toute leur valeur aux recherches détaillées que nous ne faisons ici que de proposer.

Tableau 1 Granulométrie de six échantillons de moraines latérales du bassin de Gorner

$$
\text { diamètre en } \mathrm{mm}
$$

\begin{tabular}{|c|c|c|c|c|c|c|c|}
\hline & & $\begin{array}{l}0 \\
1 \\
\stackrel{\sim}{1}\end{array}$ & $\begin{array}{l}\gamma \\
1 \\
0\end{array}$ & $\begin{array}{l}0 \\
0 \\
1 \\
\text { N }\end{array}$ & $\begin{array}{l}N^{\prime} \\
0 \\
1 \\
b_{0} \\
0\end{array}$ & $\begin{array}{l}0 \\
0 \\
0 \\
1 \\
2 \\
0\end{array}$ & $\begin{array}{l}\circ \\
\therefore \\
\text { V }\end{array}$ \\
\hline & & $\%$ & $\%$ & $\%$ & $0 / 0$ & $\%$ & $\%$ \\
\hline 1. (813) & Glacier de Grenz (Plattie) & 7 & 15 & 18 & 25 & 30 & 5 \\
\hline 2. $(815)$ & Glacier de Gorner (Ob dem See) & 8 & 18 & 22 & 32 & 20 & - \\
\hline 3. $(816)$ & Glacier de Grenz (Schwärze) & 25 & 18 & 19 & 20 & 18 & - \\
\hline 4. $(817)$ & Glacier de Gorner (Aeuss Gornerli) & 14 & 22 & 12 & 14 & 13 & 25 \\
\hline 5. $(818)$ & Glacier de Gorner (Riffelhorn) & 26 & 20 & 16 & 21 & 10 & 6 \\
\hline 6. $(820)$ & Glacier de Boden (région frontale) & 18 & 12 & 15 & 27 & 28 & - \\
\hline
\end{tabular}

\section{B I B L I O G R A P H I E}

BACKLUND, H. G.: Über die Lagerungsbedingungen eines Torffundes in NO-Grönland. Meddelelser om Grönland. 87, 1, 1931, 25 p, 3 fig. - BADER, H.: Introduction to ice petrofabrics. Journ. of Geology. 59, 6. 1951, p 519 - 536, 6 fig. - BAUER, AlB.: Glacier de Gorner; levé du mouvement des balises dans les parages du lac de Gorner. 1955. (Inédit). - BEARTh, P.: Atlas géologique de la Suisse, 1: 25 000, feuille Zermatt 535. Bern 1953. - Chamberlin, Rollin T.: Glacier movement as a typical rock deformation. Journ. of Geology. 44, 1, 1936, p 93 - 104. - DEsor, E.: Excursions et séjours dans les glaciers et les hautes régions des Alpes, de M. Agassiz et de ses compagnons de voyage. Neuchâtel 1844, XV \& 638 p, planches. - DryGalski, Erich von \& Machatscheck, Fritz: Gletscherkunde (Enzyklopädie der Erdkunde), Wien 1942. - GallowaY, R.W.: The structure of moraines in Lyngsdalen, North Norway. Journ. of Glaciology. 2, 20, 1956, p 730 - 733, 3 fig. Glen, J.W., Donner, J. J. \& West, R. G.: On the mechanism by which stones in till become oriented. Amer. Journ. of Science. 255, 1957, p 194 - 205, 4 fig. - Goldthwait, Richard P.: Development of end moraines in East-Central Baffin Island. Journ. of Geology, 59, 6, 1951, p 567 - 577, 3 fig., 3 pl. - Heim, Albert: Handbuch der Gletscherkunde. Stuttgart 1885. XVI + 560 p, 2 pl., 1 carte. Hellingworth, S. E., Carruthers, R. G., Lewis, W.V. \& King, W. B. R. : Discussion on the origin of glacial drifts. Journ. of Glaciology. 1, 8, 1950, p $430-440,3$ pl. - IvEs, J. D. \& KING, A. M.: Glaciological observations on Morsarjökull, SW. Vatnajökull. Journ. of Glaciology. 2, 16, 1954, p 423 - 428, 3 fig. - KoCH, J. P. \& Wegener, A.: Die glaciologischen Beobachtungen der Danmarkexpedition. Meddelelser om Grönland. 46, 1, 1912, 77 p, 98 fig. 5 pl. - Leighten, F. B.: Ogives of the East Twin Glacier, Alaska. Their nature and origin. Journ. of Geology, 59, 6, 1951, p $578-589$, 3 pl. - Lewis, W,V.: Discussion on the origin of glacial drifts. Journ. of Glaciology. 1, 8, 1950, p 430 - 436. - Lliboutry, L.: Etude préliminaire du glacier de Saint-Sorlin (Alpes françaises). Symposium Chamonix, 16-24 sept. 1958 - Gentbrugge 1958 -, p 45-55, 2 fig. - Meier, MARK K., Rigsby, George P. \& Sharp, Robert P.: Preliminary data from Saskatchewan Glacier, Alberta, Canada. Contribution No. 678. Division of Geol. Sciences. California Inst. of Technology (reprinted from 
"Arctic». 7, 1, 1954, p 3 - 26, 13 fig.). - Miller, Hugh: On Flexion-Structure in Till. Geol. Survey of England and Wales. Report of the British Association 1884, 1885, p 720 - 21. - Portmann, J. P.: L'orientation préférentielle des galets dans les moraines récentes du glacier de Moiry (Valais). Zeitschr. f. Gletscherkunde u. Glazialgeol. 3, 3, 1956, 313 - 316, pl. 33. - Portmann, J. P.: Les méthodes d'étude pétrographique des dépôts glaciaires. Geol. Rundschau. 45, 2, 1956, p 411 - 454. Renaud, André: Les entonnoirs du glacier du Gorner. Mémoire de la Société helv. des sc. naturelles. 71, 1, 1936, 27 p, 18 fig. - Renaud, AndrÉ: Le Bassin de Gorner: vitesse d'écoulement 1948 - 1949. 1950. (Inédit). - Renaud, André: Notice sur le glacier de Gorner (Suisse). Soc. hydrotechnique de France; tournée glaciologique, juillet 1950, 8 p, 2 pl. - Renaud, ANdRÉ: Les glaciers de la région de Zermatt in "Les dix ans du Centre alpin", Zermatt, 1955, p 40 - 46. - Russel, IsRaËL: The influence of debris on the flow of glaciers. Journ. of Geology, 3, 1895, p $823-832$. SANDFORT, K. S.: The glacial conditions and quaternary history of North East Land. Geogr. Journ. 74, 5 - 6, 1929, p $451-552,3$ fig. - ShARP, RoBerT P.: Studies of superglacial debris on valley glaciers. Am. Journ. of Sc. 247, 5, 1949, p $289-315$. - Untersteiner, N.: Some observations on the banding of glacier ice. Journ. of Glaciology. 2, 17, p $502-506,4$ fig. - WARD,W. H.: The physics of deglaciation in Central Baffin Island. Journ. of Glaciology. 2, 11, 1952, p $9-22,11$ fig. Washburn, A. L.: Unusual patterned Ground in Greenland. Bull. geol. Soc. Am. 67, 6, 1956, p $807-810,2 \mathrm{pl}$. - WASHBURN, A. L.: Reconnaissance geology of portions of Victoria Island and adjacent regions, Arctic Canada. Memoir Geol. Soc. Amer. 22, 1947, 142 p, 4 fig. 32 pl.

\title{
GESTEINSEINSCHLÜSSE IN GLETSCHERN
}

Die vorliegenden Untersuchungen entspringen der Tatsache, daß der Gletscher einen Komplex darstellt, in welchem verschieden große Gesteinsfragmente in einer Grundmasse, dem Eis, festgehalten sind. Im allgemeinen wird nur dem Eis Beachtung geschenkt. Es ist anzunehmen, daß ein auch nur schwacher Gehalt an erratischem Material die physischen Eigenschaften des Eises verändern kann. Gestützt auf Angaben in einigen Publikationen zeigt der Verfasser den Einfluß der Gesteinseinschlüsse auf die Plastizität, die Art der Deformation und die Ablationsstärke des Gletschers auf. Es wäre wichtig, das Verhältnis der Gesteinskomponenten, ihre Korngröße und mineralogische Natur, sowie ihre Form und Richtung festzustellen, um ihre Verteilung im Eis und den Transportvorgang kennen zu lernen.

Um ein Beispiel anzuführen, weist der Verfasser auf einige Beobachtungen hin, die er auf dem Gornergletscher ob Zermatt (Schweiz) machte, wo die Richtung der Hauptachse der in der Gletscheroberfläche eingelegten Geschiebe gemessen wurde. Es kann eine Vorzugsrichtung festgestellt werden (Fig. 2). Sie hängt von der Gestaltung des Gletschers ab, auch wenn die Beziehungen zu dessen Struktur nicht immer deutlich erkennbar sind. Ferner erwähnt der Verfasser die Richtung der Hauptachse der Gesteinskomponenten in Seitenmoränen (Fig. 3). Schließlich wird die Korngröße einiger aus Moränen stammenden Proben bestimmt im Zusammenhang mit ihrer petrographischen Natur und jener der Gesteine in situ. Die verschiedenen Probleme werden mit diesen ersten Beobachtungen nicht erschöpfend behandelt; die letzteren mögen aber zu systematischer Forschung anregen.

\section{DANIEL MEYER, EIN UNBEKANNTER SCHWEIZERISCHER KARTOGRAPH UND DER KATASTER SEINER ZEIT}

\author{
Franz Grenacher
}

Über das Leben, verdienstvolle Wirken und die Bedeutung der bekannten Lohnherren und Kartographen Jakob Meyer (1614-1678) und Sohn Georg Friedrich Meyer (1645-1693) sind wir durch Karten, Pläne, Akten und neuzeitliche Publikationen ${ }^{1}$ reichlich unterrichtet. Hingegen ist noch unbekannt, da $\beta$ einem weiteren Glied

${ }^{1}$ F. Burckhardt: Über Pläne und Karten des Baselgebietes aus dem 17. Jahrhdt. in Basler Zeitschr. f. Altertumskde. u. Geschichte, 1906, S. 291 - 360. R. Wolf: Geschichte der Vermessung der Schweiz, S. 37 -40, Zürich 1879. R. GroB: Geschichte der schweiz. Kartographie, S. 41-42, Bern 1941. P. SUTER: Georg Friedrich Meyer, ein Basler Kartograph des 17. Jahrhunderts, Schweizer Geograph, 1933. F. GrenaChER: Die Anfänge der Militärkartographie am Oberrhein, Basler Ztschr. 1958, S. 97-100. 